\title{
Clinical expertise and reasoning with uncertain categories
}

\author{
Brett K. Hayes and Tsan-Hsiang Jessamine Chen \\ University of New South Wales, Sydney, New South Wales, Australia
}

\begin{abstract}
Expert clinical psychologists, clinical psychology graduates, and nonclinical graduate students were presented with clinical and nonclinical cases in which the diagnosis or category membership of a character was uncertain; they then made feature predictions about the character. For each case, there was a diagnosis or category that was highly probable and a less likely alternative that either did (relevant condition) or did not (neutral condition) alter predictions. For clinical cases, clinical experts and graduate clinicians gave different predictions for the relevant and neutral conditions, indicating that they had considered the uncertain nature of the diagnosis in their predictions. Although they acknowledged that the diagnosis was uncertain, nonclinical students ignored the less likely diagnostic alternatives when making predictions. For the nonclinical cases, all three groups made predictions based on only the most likely category alternative. The results showed that clinical training and/or experience promote multiple-category reasoning, but that this effect is domain specific.
\end{abstract}

Category-based induction is inherently probabilistic, and new observations increase or decrease confidence in the belief that a property generalizes across category members. Murphy and Ross (1994) highlighted an additional complexity in category-based inference: Predictions may have to be made about objects whose category membership has yet to be determined. For example, a patient's symptoms may be consistent with a number of alternative diagnoses, but predictions about the future course of their illness may have to be made before a specific diagnosis has been reached.

An important question, therefore, is how people make inferences when category membership is uncertain. One answer comes from models that assume that induction follows Bayesian principles. According to Anderson's (1991) rational model, people estimate the probabilities of a predicted property for each category alternative and combine these estimates. Malt, Ross, and Murphy (1995) tested this prediction by presenting undergraduates with stories about a character who was most likely a realtor, but who may have belonged to a different category (cable $\mathrm{TV}$ technician or burglar). The predicted probability of a future behavior (the man ringing the doorbell) was lower when the category alternative was "burglar" than when it was "technician." According to the Bayesian prescription, people consider multiple categories and their predictions will differ across these conditions. Malt et al., however, found that predictions were based on a consideration of only the most probable category, and that subjects ignored less certain alternatives. Such "single-category reasoning" has been found in a range of induction tasks that utilize real-world and artificial categories (see, e.g., Murphy \& Ross, 1994; Ross \& Murphy, 1996).
One important extension of this work has been the study of predictions about objects that belong to more than one category (e.g., muffins are both "breads" and "breakfast foods"). Predictions of the probability that such an object has a particular property can vary depending on which category is considered. Although Anderson's (1991) model does not apply in this case, related Bayesian approaches (e.g., Tenenbaum, Kemp, \& Shafto, 2007) suggest that the multiple categories to which an instance belongs should be considered in property inference. Murphy and Ross (1999), however, found that people usually make predictions about cross-classified objects based on only the category judged to be the most appropriate description in a given context. Moreover, single-category reasoning was equally prevalent for cross-classified objects and for those whose category membership was uncertain.

Although there appears to be broad support for the prevalence of single-category reasoning, it is interesting to consider whether it is affected by expertise when one makes inferences with multiple (uncertain or crossclassified) categories. Domain expertise could facilitate multiple-category reasoning in a number of ways. Expertise promotes the detection of more coherent or meaningful patterns in familiar stimuli, allowing for the encoding of larger chunks of information (Chase \& Simon, 1973). Hence, experts might encode the relevant features from more probable categories more efficiently than novices might, freeing up cognitive resources for the consideration of less likely alternatives.

Expertise is also associated with greater flexibility in inferential reasoning. In reasoning about biological kinds, novices usually infer novel properties, largely on the basis of taxonomic similarity, whereas experts consider a range

B. K. Hayes, b.hayes@unsw.edu.au 
of relations, including ecological and causal links (Proffitt, Coley, \& Medin, 2000; Shafto \& Coley, 2003). Inferential flexibility, however, is specific to stimuli that are familiar to experts. Shafto and Coley found that expert fishermen considered a range of relations when generalizing a familiar property but considered only taxonomic relations when generalizing novel properties. Inferential flexibility could facilitate multiple-category reasoning by promoting attention to a broader range of associations between a to-be-predicted property and multiple relevant categories. Ross and Murphy (1996) have shown that multiple-category reasoning is more common when people see clear associations between a nontarget category and the feature being predicted.

A more pragmatic reason why expertise may promote multiple-category reasoning is that experts are often forced to consider the consequences of uncertain categorization and in some cases are trained to do so. Making predictions under uncertainty is a challenge that experts face daily in domains such as clinical psychology, medicine, and economics. The neglect of category alternatives is also likely to have more serious consequences in these domains than in many laboratory studies. In the Murphy and Ross (1994) experiments, for example, the additional effort involved in considering category alternatives led only to small changes in feature predictions $(10 \%-15 \%)$. A failure to consider alternative clinical diagnoses, however, could lead to serious errors in treatment planning. For this reason, clinical training programs caution against premature diagnosis and emphasize the consideration of diagnostic alternatives (see, e.g., Antony \& Barlow, 2002). Explicit consideration of alternative hypotheses can reduce unhelpful biases, such as seeking confirmatory information (see Koehler, 1991, for a review). By extension, training that emphasizes the consideration of uncertain alternatives could facilitate multiple-category reasoning.

Nevertheless, domain experts are prone to reasoning biases, and these may have an impact on multiple-category reasoning. Clinical experts often show overconfidence in initial diagnoses (Arkes, 1981), which can lead to an increased focus on the most probable diagnostic category. Hence, it was important in the present study to compare expert and novice confidence in category identification and examine its effects on multiple-category reasoning.

The main aims of the present study, therefore, were to examine whether domain expertise promotes multiplecategory reasoning and whether such effects are domain specific. The target domain was clinical psychology. This domain was chosen because, although it uses nosological systems with clear diagnostic guidelines, in practice diagnosis is often based on incomplete and ambiguous evidence (Kim \& Ahn, 2002). The effect of expertise was tested in two ways. First, we compared multiple-category reasoning in clinical cases by trained clinicians and by psychologists who had no clinical training. Second, we examined multiple-category reasoning within subjects by comparing inferences about clinical and nonclinical cases.

A subsidiary question was whether multiple-category reasoning is affected by level of expertise. Past com- parisons of more expert and less expert clinicians have revealed a range of differences in their memory for and reasoning about case materials (Norman, 2005). For example, highly experienced clinical psychologists are more likely to perceive overlaps between psychodiagnostic categories (Murphy \& Wright, 1984). They therefore may be more likely to activate multiple relevant categories for a given clinical instance, leading to more multiple-category inferences.

Expert clinical psychologists, clinical graduate students, and nonclinical graduates were shown clinical and nonclinical cases. In each case, one target category was clearly the most probable, but less likely, plausible category alternatives were also present. The impact of these alternatives on feature predictions was varied between participants. According to a Bayesian perspective, in the prediction neutral condition, consideration of a nontarget category will not alter the probability of a critical feature, but in the prediction relevant condition, consideration of a nontarget category will alter feature probability. Hence, multiple-category reasoning was indexed by differences in feature probability ratings between relevant and neutral conditions.

\section{METHOD}

\section{Participants}

Clinical experts were 32 clinical psychologists with an average of 12 years of experience following graduate training $(S D=5.95$, range $=5-30$ years), recruited from a metropolitan hospital and community clinics. Clinical novices were 36 psychology graduates who had recently completed or who were about to complete graduate clinical training (a two-year professional masters degree, with approximately $800 \mathrm{~h}$ of patient contact).

Nonclinicians were 30 psychology graduate students enrolled in nonclinical programs (e.g., PhD in cognitive science). Entry-level grade point averages for these programs were equal to or greater than those required for the clinical programs. A majority of nonclinicians $(58 \%)$ had completed introductory courses in psychopathology, but none had graduate training in this field.

A further 13 clinical experts ( $M=10$ years of experience), 14 graduate clinical psychology students, and 30 nonclinical doctoral students took part in pilot testing.

\section{Construction of Case Vignettes}

There were two clinical cases (one child disorder and one adult disorder) and one nonclinical case, each of which had two versions (neutral and relevant). Case structure is summarized in Table 1. Cases were of similar length (294-330 words). In the child case, mental retardation was the target diagnosis (nine symptoms consistent with this diagnosis were mentioned). The nontarget category alternative was autistic spectrum disorder in the neutral condition and attention deficit hyperactivity disorder (ADHD) in the relevant condition. The alternative category was introduced early in the case description as part of the psychiatric history and was highlighted again at the end (see the Appendix). Pilot testing with clinical graduates and experts $(n=27)$ confirmed that the rated probability of a critical symptom ("will show self-injurious behaviors") was similar when the only diagnosis presented was mental retardation $(M=.48)$ or autism $(M=.47)$, but was lower when the diagnosis was ADHD $(M=.23)[F(1,26)=32.1, p<$ $\left..001 ; \eta_{\mathrm{p}}^{2}=.71\right]$. Pilot nonclinicians $(n=19)$ also showed no difference in critical symptom probabilities for mental retardation $(M=$ $.35)$ and autism $(M=.43)$, but gave a lower probability rating for 
Table 1

Summary of the Case Design

\begin{tabular}{|c|c|c|c|c|c|c|}
\hline & \multicolumn{2}{|c|}{ Child Clinical } & \multicolumn{2}{|c|}{ Adult Clinical } & \multicolumn{2}{|c|}{ Nonclinical } \\
\hline & $\begin{array}{l}\text { Prediction } \\
\text { Neutral }\end{array}$ & $\begin{array}{c}\text { Prediction } \\
\text { Relevant }\end{array}$ & $\begin{array}{l}\text { Prediction } \\
\text { Neutral }\end{array}$ & $\begin{array}{c}\text { Prediction } \\
\text { Relevant }\end{array}$ & $\begin{array}{l}\text { Prediction } \\
\text { Neutral }\end{array}$ & $\begin{array}{l}\text { Prediction } \\
\text { Relevant }\end{array}$ \\
\hline Target category & MR & $\mathrm{MR}$ & PTSD & PTSD & realtor & realtor \\
\hline Nontarget category & autism & ADHD & GAD & MDD & $\begin{array}{l}\text { cable TV } \\
\text { technician }\end{array}$ & burglar \\
\hline $\begin{array}{l}\text { Critical feature } \\
\text { prediction }\end{array}$ & $\begin{array}{l}\text { "likelihood that } \\
\text { they will show } \\
\text { self-injurious } \\
\text { behaviors" }\end{array}$ & $\begin{array}{l}\text { "likelihood that } \\
\text { they will show } \\
\text { self-injurious } \\
\text { behaviors" }\end{array}$ & $\begin{array}{l}\text { "likelihood that } \\
\text { they will expe- } \\
\text { rience jittery } \\
\text { feelings for } \\
\text { some time" }\end{array}$ & $\begin{array}{l}\text { "likelihood that } \\
\text { they will expe- } \\
\text { rience jittery } \\
\text { feelings for } \\
\text { some time" }\end{array}$ & $\begin{array}{l}\text { "likelihood that } \\
\text { they will ring } \\
\text { the doorbell } \\
\text { within the next } \\
15 \text { minutes" }\end{array}$ & $\begin{array}{l}\text { "likelihood that } \\
\text { they will ring } \\
\text { the doorbell } \\
\text { within the next } \\
15 \text { minutes" }\end{array}$ \\
\hline
\end{tabular}

Note-MR, mental retardation; PTSD, posttraumatic stress disorder; ADHD, attention deficit hyperactivity disorder; GAD, generalized anxiety disorder; MDD, major depressive disorder.

ADHD $(M=.29)$. The latter difference approached significance $\left[F(1,18)=4.71, p=.08 ; \eta_{\mathrm{p}}^{2}=.21\right]$

The adult clinical case had an identical structure but described a male adult suffering emotional problems after trauma. Posttraumatic stress disorder (PTSD) was the target diagnosis. The nontarget alternative was generalized anxiety disorder (GAD) in the neutral condition and major depressive disorder (MDD) in the relevant condition. The critical feature was the likelihood that the adult patient "will experience jittery feelings." Pilot testing with clinical graduate students and experts confirmed that the probability of this feature was similar when the diagnosis was $\operatorname{PTSD}(M=.77)$ or $\operatorname{GAD}(M=$ $.75)$, but lower when it was depression $(M=.34)[F(1,26)=64.24$, $\left.p<.001 ; \eta_{\mathrm{p}}^{2}=.55\right]$. Again, nonclinicians showed a similar pattern, with similar probabilities for $\operatorname{PTSD}(M=.67)$ and $\operatorname{GAD}(M=.78)$ but lower ratings for depression $(M=.28)[F(1,18)=60.43, p<$ $\left..001 ; \eta_{\mathrm{p}}^{2}=.77\right]$.

The nonclinical case was based on Malt et al. (1995) and described an unfamiliar character walking up a driveway. The character's target occupation was "realtor." The nontarget alternative was "cable TV technician" in the neutral condition and "burglar" in the relevant condition. Again, the critical feature ("likelihood that they will ring the doorbell") was such that consideration of the nontarget category would lower feature probability in the relevant condition but not in the neutral condition.

\section{Comorbidity Ratings}

To examine whether clinical categories were perceived as mutually exclusive, pilot expert and novice clinicians $(n=27)$ and nonclinicians $(n=30)$ rated the probability that each of the four pairs of target and alternate diagnoses could "co-occur in a clinical sample" $(0=$ low chance $; 100=$ high chance $)$. The probability of comorbidity was judged low to moderate $(M=35.05)$ and, in general, did not vary with level of expertise. The one exception was PTSD and depression, for which experts viewed comorbidity as more likely $(M=$ $52.08)$ than did novices or nonclinicians $(M=41.08)[F(1,56)=$ $\left.3.99, p<.05 ; \eta_{\mathrm{p}}^{2}=.13\right]$. Note that even though the target and nontarget instances were viewed as members of nonexclusive categories, it was still reasonable to ask whether both categories were considered in property inference (cf. Murphy \& Ross, 1999).

\section{Procedure}

Equal numbers of nonclinical students, clinical novices, and experts were randomly allocated to neutral or relevant conditions. Participants read and then answered questions about adult and child clinical cases in counterbalanced order, followed by the nonclinical case. This presentation order was used to eliminate a possible elevation of multiple-category reasoning in the clinical domain as a result of prior completion of a nonclinical item.

After reading each case, participants turned a page and answered six questions. These included the critical feature prediction, made on a 100 -point scale $(0=$ not at all likely, $100=$ highly likely $)$.
Participants also judged and rated their certainty about "the most likely diagnosis/occupation" ( $0=$ not at all certain, $100=$ very certain). These target-category questions always followed feature predictions. Prediction and categorization questions were interspersed with three items that involved recall of case details. Experts were tested individually at their workplace, or they returned completed questionnaires by mail. Novice clinicians and nonclinical students were tested individually or in small groups.

\section{RESULTS}

A preliminary analysis confirmed that recall of case details was accurate (mean proportion correct $=.9$ ) and did not vary with case, expertise, or relevance.

\section{Identification of the Target Category}

Our predictions were based on the assumption that people would identify the intended target category for each case. To check this, we examined rates of target category identification. Confidence ratings for these judgments were also taken in order to assess whether category membership was viewed as uncertain.

For the child clinical case, the intended target was correctly identified on most occasions $(65 \%)$, and this did not vary with level of expertise. Target identification was more accurate in the relevant condition $(M=84 \%)$ than in the neutral condition $(M=46 \%)[F(1,92)=20.18$, $\left.p<.001 ; \eta_{\mathrm{p}}^{2}=.18\right]$. Although the latter difference was unexpected, it did not alter the predicted probability of the critical feature in the neutral condition, because pilot testing showed that this was the same for target and nontarget categories. Confidence in target identification was moderate $(M=60.46)$ and did not vary with level of expertise or relevance $(F \mathrm{~s}<1.8)$.

For the adult clinical case, the target category was correctly identified on most occasions $(M=95 \%)$, regardless of level of expertise or relevance $(F \mathrm{~s}<1.5)$. Confidence in target identification varied with level of expertise. Clinical experts $(M=79.0)$ and novices $(M=70.43)$ had similar confidence levels, but both were more confident than nonclinicians $(M=63.39)[F(1,87)=7.32, p<$ $\left..001 ; \eta_{\mathrm{p}}^{2}=.08\right]$.

For the nonclinical case, the target category was correctly identified on most occasions (experts, $M=86 \%$; novices, $M=84 \%$; nonclinicians, $M=88 \%$ ), regardless 
of level of expertise or relevance (all $F$ s $<2.0$ ). Confidence in identification was moderate $(M=60.66)$ and did not vary with level of expertise or relevance.

Hence, with one exception, cases were identified as most likely being exemplars of the target category. Notably, though, moderate confidence judgments showed that category membership in each case was viewed as uncertain.

\section{Predictions About Critical Features}

If multiple categories were used, then we expected probability ratings for the critical feature to differ in the relevant and neutral conditions. Note, though, that this follows only if the target category was identified correctly in the relevant condition. Hence, for this condition, only predictions from those who correctly identified the target were included in the analysis. Predicted probabilities for critical features are given in Table 2 . These data were entered into 3 (expertise) $\times 2$ (prediction relevance) ANOVAs with planned contrasts on the expertise factor.

For the child clinical case, clinicians rated the critical feature as more likely than did nonclinicians $[F(1,75)=$ $\left.5.61, p<.05 ; \eta_{\mathrm{p}}^{2}=.07\right]$. Probability ratings were higher in the neutral condition than in the relevant condition $\left[F(1,75)=4.46, p<.05 ; \eta_{\mathrm{p}}^{2}=.06\right]$. Critically, these main effects were moderated by an interaction between relevance and the contrast comparing clinicians with nonclinicians $\left[F(1,75)=3.92, p<.05 ; \eta_{\mathrm{p}}^{2}=.05\right]$. Clinicians rated the critical feature as less probable in the relevant condition than in the neutral condition, whereas nonclinicians gave similar ratings for each condition (see Table 2). One concern was that the neutral baseline probability for nonclinicians was lower than expected, given clinicians' pilot symptom ratings, and lower than the neutral baseline probabilities given by clinicians. Inspection of individual data suggested that this occurred because 2 nonclinicians responded with a zero-probability rating. When those responses were removed, the mean probability for the nonclinical neutral group increased to 32.7. This mean did not differ reliably from the neutral baseline estimates given by clinicians. Critically, this revised estimate also did not differ from the probability ratings given by nonclinicians in the relevant condition $(p=.6)$. Hence, both groups of clinicians - but not the nonclinicians - showed multiplecategory reasoning for this case.

For the adult clinical case, there were no main effects of level of expertise or relevance on feature probability. There was a significant interaction between relevance and the contrast comparing the two clinician groups
$\left[F(1,90)=6.1, p<.01 ; \eta_{\mathrm{p}}^{2}=.06\right]$. Novice clinicians gave lower probability ratings in the relevant condition than in the neutral condition, whereas experts showed the opposite trend. Nonclinicians gave similar ratings for both conditions. Only expert and novice clinicians considered nontarget categories during feature inference. Interestingly, though, this affected their probability estimates in different ways.

For the nonclinical case, there was no difference in probability ratings for the two relevance conditions for any group, and no significant interactions (all $F \mathrm{~s}<1.6$ ).

\section{DISCUSSION}

This study examined the effects of expertise on singleand multiple-category reasoning in clinical and nonclinical domains. Previous work had suggested that when category membership is uncertain or when an object belongs to multiple categories, people ignore information about less likely alternatives and base their feature predictions on information from only the most likely category (Murphy \& Ross, 1994, 1999).

Graduate psychologists with no clinical training performed in a manner consistent with past research. Nonclinicians recognized that category membership was uncertain but always used single-category reasoning for feature predictions. Expert and graduate clinical psychologists, however, showed clear evidence of multiple-category reasoning, with less likely category alternatives affecting judgments about the probability of clinical symptoms. Hence, clinical expertise promoted multiple-category reasoning for familiar stimuli.

Our general expectation was that, for clinical cases, consideration of a nontarget category would lower probability ratings for critical symptoms. This was confirmed in three out of four clinical cases (the child and adult cases for clinical students, and the child case for experts). In the adult case, unexpectedly, consideration of the nontarget category led to an increase rather than a decrease in experts' ratings. The reason for this unexpected finding is not entirely clear, but a possible explanation involves differences between expert and novice beliefs about comorbidity, together with an indication that experts saw causal links between diagnoses. Pilot comorbidity ratings suggested that experts in the relevant condition were more likely to view target (PTSD) and nontarget (depression) diagnoses as comorbid. During debriefing, many experts in this condition also endorsed the view that these disorders were causally linked, citing evidence that comorbid depression can exacerbate PTSD

Table 2

Mean Probability Ratings (\%, With Standard Deviations) for Critical Feature Predictions

\begin{tabular}{|c|c|c|c|c|c|c|c|c|c|c|c|c|}
\hline \multirow[b]{3}{*}{ Expertise Level } & \multicolumn{4}{|c|}{ Child Clinical } & \multicolumn{4}{|c|}{ Adult Clinical } & \multicolumn{4}{|c|}{ Nonclinical } \\
\hline & \multicolumn{2}{|c|}{$\begin{array}{c}\text { Prediction } \\
\text { Neutral }\end{array}$} & \multicolumn{2}{|c|}{$\begin{array}{c}\text { Prediction } \\
\text { Relevant }\end{array}$} & \multicolumn{2}{|c|}{$\begin{array}{c}\text { Prediction } \\
\text { Neutral }\end{array}$} & \multicolumn{2}{|c|}{$\begin{array}{c}\text { Prediction } \\
\text { Relevant }\end{array}$} & \multicolumn{2}{|c|}{$\begin{array}{c}\text { Prediction } \\
\text { Neutral }\end{array}$} & \multicolumn{2}{|c|}{$\begin{array}{c}\text { Prediction } \\
\text { Relevant }\end{array}$} \\
\hline & $M$ & $S D$ & $M$ & $S D$ & $M$ & $S D$ & $M$ & $S D$ & $M$ & $S D$ & $M$ & $S D$ \\
\hline Nonclinical students & 26.79 & 16.36 & 29.67 & 19.41 & 73.67 & 12.60 & 73.00 & 21.03 & 79.00 & 21.89 & 75.67 & 16.24 \\
\hline Novice clinicians & 43.53 & 16.18 & 29.58 & 19.12 & 73.75 & 9.57 & 63.68 & 16.41 & 70.63 & 17.11 & 67.12 & 20.90 \\
\hline Expert clinicians & 46.67 & 19.70 & 32.86 & 16.84 & 70.00 & 16.41 & 79.41 & 17.13 & 68.08 & 23.85 & 76.11 & 17.03 \\
\hline
\end{tabular}


symptoms (O’Donnell, Creamer, \& Pattison, 2004). This could explain why experts believed that the joint occurrence of depression and PTSD increased the likelihood of the critical symptom. Note that such "causal" multiplecategory reasoning represents a form of induction that is qualitatively different from the Bayesian approaches that were described by Murphy and Ross (1994).

Our second aim was to examine whether the effects of clinical expertise generalized to inferences under uncertainty in nonclinical contexts. Like previous studies of expertise on induction in folk biology (e.g., Shafto \& Coley, 2003), our study yielded little evidence for such a generalization. Clinicians who used multiple-category reasoning in clinical cases reverted to single-category reasoning in the nonclinical case.

A potential problem with expert reasoning is diagnostic overconfidence, which can potentiate single-category inference. Our clinicians were more confident than nonclinicians in classifying adult clinical cases. This increased confidence, however, did not prevent them from considering multiple categories when making predictions. As long as clinicians viewed category membership as somewhat uncertain (expert confidence in category identification was below ceiling), they appeared to consider multiple categories when making clinical inferences.

\section{Implications for Theories of Expertise and Multiple-Category Reasoning}

One explanation for single-category reasoning is that consideration of uncertain categories and conditional feature probabilities may exceed working memory capacity. Alternately, people may not understand the conditional nature of problems involving uncertainty, failing to realize that category uncertainty might have an impact on subsequent predictions. According to this view, singlecategory reasoning resembles other failures of normative reasoning, such as base-rate neglect in the estimation of event probability.

The data in the present study do not support such accounts. The academic entry level for the nonclinical graduates was as high or higher than for clinicians. Hence, clinicians were unlikely to have better working memories than did nonclinicians. Moreover, if single-category reasoning was due to a general neglect of category alternatives, it is difficult to see why this would have varied across domains.

Our findings are better explained by considering the known effects of domain expertise on memory and reasoning, and how these affect multiple-category reasoning. Experts' more efficient encoding of familiar features and categories may have facilitated their consideration of multiple categories during inference. The results are also consistent with findings that experts have a better understanding of the multiple relations between familiar features and categories and apply this knowledge more flexibly (see, e.g., Shafto \& Coley, 2003). Previous work, however, has been concerned with property inference in instances for which category membership is known. The present study suggests that expertise-based increases in inductive flexibility extend to cases in which category membership is uncertain.
It is also possible that aspects of professional training in clinical psychology, such as instruction in the consideration of multiple diagnoses, may have boosted multiplecategory reasoning for familiar case materials. This is suggested by the similarity in the expertise effects found for graduate and expert clinicians. Teasing apart the relative contributions of domain experience and training in the facilitation of multiple-category reasoning is an important goal for future work.

Previous work on inferences under category uncertainty has suggested that people often use a single-category heuristic, ignoring less likely category alternatives when framing predictions. We have shown that experts can overcome this heuristic when making inferences about instances of familiar categories cases. Single-category reasoning, therefore, may be prevalent only in inferences about unfamiliar domains and in which neglect of category alternatives has only a modest impact on the prediction.

\section{AUTHOR NOTE}

This work was supported by Australian Research Council Discovery Grant DP0770292 to B.K.H. The authors thank all of the participants for their enthusiastic involvement in this study. We also thank Gregory Murphy, Michelle Moulds, and John Coley for their valuable comments. Correspondence concerning this article should be addressed to B. K. Hayes, School of Psychology, University of New South Wales, Sydney, NSW 2052, Australia (e-mail: b.hayes@unsw.edu.au).

\section{REFERENCES}

ANDERSON, J. R. (1991). The adaptive nature of human categorization. Psychological Review, 98, 409-429.

Antony, M. M., \& BARLow, D. H. (EDs.) (2002). Handbook of assessment and treatment planning for psychological disorders. New York: Guilford.

ArKes, H. R. (1981). Impediments to accurate clinical judgment and possible ways to minimize their impact. Journal of Consulting \& Clinical Psychology, 49, 323-330.

Chase, W. G., \& Simon, H. A. (1973). Perception in chess. Cognitive Psychology, 4, 55-81.

KIM, N. S., \& AHN, W.-K. (2002). Clinical psychologists' theory-based representations of mental disorders predict their diagnostic reasoning and memory. Journal of Experimental Psychology: General, 131, 451-476.

Koenler, D. J. (1991). Explanation, imagination, and confidence in judgment. Psychological Bulletin, 110, 499-519.

Malt, B. C., Ross, B. H., \& Murphy, G. L. (1995). Predicting features for members of natural categories when categorization is uncertain. Journal of Experimental Psychology: Learning, Memory, \& Cognition, 21, 646-661.

Murphy, G. L., \& Ross, B. H. (1994). Predictions from uncertain categorizations. Cognitive Psychology, 27, 148-193.

MurPhy, G. L., \& Ross, B. H. (1999). Induction with cross-classified categories. Memory \& Cognition, 27, 1024-1041.

MurPhy, G. L., \& Wright, J. C. (1984). Changes in conceptual structure with expertise: Differences between real-world experts and novices. Journal of Experimental Psychology: Learning, Memory, \& Cognition, 10, 144-155.

Norman, G. (2005). Research in clinical reasoning: Past history and current trends. Medical Education, 39, 418-427.

O’Donnell, M. L., Creamer, M., \& Pattison, P. (2004). Posttraumatic stress disorder and depression following trauma: Understanding comorbidity. American Journal of Psychiatry, 161, 1390-1396.

Proffitt, J. B., Coley, J. D., \& Medin, D. L. (2000). Expertise and category-based induction. Journal of Experimental Psychology: Learning, Memory, \& Cognition, 26, 811-828.

Ross, B. H., \& MurPhy, G. L. (1996). Category-based predictions: Influence of uncertainty and feature associations. Journal of Experimental Psychology: Learning, Memory, \& Cognition, 22, 736-753. 
Shafto, P., \& Coley, J. D. (2003). Development of categorization and reasoning in the natural world: Novices to experts, naive similarity to ecological knowledge. Journal of Experimental Psychology: Learning, Memory, \& Cognition, 29, 641-649.
Tenenbaum, J. B., Kemp, C., \& Shafto, P. (2007). Theory-based Bayesian models of inductive reasoning. In A. Feeney \& E. Heit (Eds.), Inductive reasoning: Experimental, developmental, and computational approaches (pp. 167-204). Cambridge: Cambridge University Press.

\section{APPENDIX}

In the following two examples, text included in the prediction-neutral condition is given in italics; text included in the prediction-relevant condition is given in bold.

\section{Child Clinical Case Description}

Megan is a 13-year-old girl who began attending special education classes at age three, though she and her family began receiving support services shortly after her birth. In her case history, another clinician mentioned the possibility of autistic disorder. She has an unusual ability to memorize and is fascinated with reading, even though she appears not to comprehend anything she reads. In her case history, another clinician mentioned the possibility of Attention Deficit/Hyperactivity Disorder (ADHD). According to her class teacher, Megan is hardly ever in her seat, but roams around the class, talking to other children while they are working. Megan's learning skills have been evaluated a number of times over the years, with her I.Q. scores ranging from the upper 30 s to the mid $40 \mathrm{~s}$. Her most recent evaluation resulted in a mental age of five years and seven months, and an I.Q. score of 46. During Kindergarten through Year 6, she was integrated into a regular classroom for part of each school day. Her class teacher observed that she often held her hands in her pockets and spun around in place.

The two major targets of the educational programs Megan has received are development of self-help skills and functional academics. Although Megan's results from the AAMR Adaptive Behavior Scale indicated an age-equivalent performance of six years, two months, Megan is able to dress and groom herself and can perform various household chores such as laundry and vacuuming. She can count, make change, and is able to do basic addition and subtraction. She reads and spells at about a Year 2 to Year 3 level. Megan is currently receiving job training skills as part of the transition services provided by her school district. Her parents are optimistic that she may eventually be able to obtain semi-independent employment. If not, they hope she will be employed at a sheltered workshop. However, they worry about her poor social skills and that she becomes extremely upset when there are changes in her environment. However, they worry about her difficulty to keep her attention on tasks and that she becomes distracted easily.

\section{Adult Clinical Case Description}

Mr. Roberts, a 37-year-old Irish fireman, was hospitalized for second- and third-degree burns over a third of his body. [Mr. Roberts' case history indicated diagnosis of general anxiety disorder in his early 30's in that he constantly worried about whether he was a good father to his children and whether he was liked by co-workers on the job.] / [Mr. Roberts' case history indicated episodes of depression in his early 30's where for several months he had trouble concentrating on his job and had depressed moods.] During the month he spent on the burn unit, he was the model "stoic patient," but a week after discharge, during his first appointment at the surgical clinic, he was tremulous, stammering, and unresponsive to the surgeon's assurances. The surgeon paged the burns unit consultant-liaison psychologist and introduced him to Mr. Roberts. Although Mr. Roberts tried to appear confident on assessment, he chain-smoked, glanced around furtively, squirmed in his chair, and at times burst into tears. When he was able to calm down, he explained that he could not stop thinking about how, for the first time in his distinguished career, he entered a burning building alone, in a manner contrary to the safety procedure he was responsible for teaching, and sustained near-fatal burns.

During his hospitalization he was troubled by frequent terrible nightmares about the fire. He did not say anything about them because he thought they would pass. When he returned home, he had recurrent nightmares, in which he re-experiences the fire over and over again. He became constantly jumpy and nervous and drunk wine to calm his nerves and to sleep. He felt humiliated about his mistake at the fire and could not stop replaying it in his mind. He was very ashamed about having to face his co-workers in his present condition - shaky, sweating, and frightened. He was scheduled to return to his duties on a part-time basis in two weeks, but he did not think he ever will be able to stand going back to the fire station or going out to fight a fire again. The burns unit consultantliaison psychologist suspected that he had developed symptoms of [generalized anxiety] / [depression].

(Manuscript received December 20, 2007; revision accepted for publication April 16, 2008.) 